\title{
Triplet State Formation Along the Ultrafast Decay of Excited Singlet Cytosine
}

\author{
by \\ Manuela Merchán, Luis Serrano-Andrés, \\ Michael A. Robb, and Lluís Blancafort
}

\section{Supporting Information}

\section{Additional Computational Details.}

Calculation of critical points and minimum-energy paths. The calculation of the critical points and MEP on the singlet state surface has been reported in previous publications. ${ }^{1-3}$ Structures ${ }^{3}\left(\pi, \pi^{*}\right)_{\min }$ and $\left(\mathbf{T}_{\mathbf{1}} / \mathbf{S}_{\mathbf{0}}\right)_{\mathbf{X}}$, as well as all reported MEP, were optimized at the CASSCF $(8,7)$ level of theory with the $6-31 \mathrm{G}(\mathrm{d}, \mathrm{p})$ or $6-31 \mathrm{G}(\mathrm{d})$ basis, using the algorithms implemented in Molcas- $6{ }^{4}$ and Gaussian03. ${ }^{5}$ The active space used for the optimizations comprises eight active $\pi$ electrons distributed in seven $\pi$ MOs. The $\pi$ MO localized on the $\mathrm{NH}_{2}$ moiety was kept inactive. We note that the additional hydrogen polarization functions of the 6-31G(d) have only a small effects on the structures and relative energies. ${ }^{2}$ Structure ${ }^{3}\left(\mathbf{n}_{\mathbf{O}}, \pi^{*}\right)_{\text {min }}$ was optimized using a $(14,10)$ active space $\left(8 \pi+n_{\mathrm{O}}+n_{N}\right.$, 2nd root of four-root stateaveraged calculation), and ${ }^{3}\left(\mathbf{n}_{\mathbf{N}}, \pi^{*}\right)_{\min }$ using a $(10,8)$ active space $\left(7 \pi+n_{N}\right.$, 2nd root of three-root stateaveraged calculation).

Triplet minima and $S_{1}$ MEP from FC to ${ }^{1}\left(\pi, \pi^{*}\right)_{\min }$. The Cartesian coordinates for the optimized triplet state structures are listed in Table SI1 and the main geometrical parameters for the low-lying singlet and triplet state minima are shown in Figure SI1. Starting from the FC geometry, ${ }^{2}$ the MEP was built by imposing the constraint in the 
hypersphere radius of 0.01 au (fifteen structures). The $\operatorname{CASSCF}(12,9) / 6-31 G(d, p)$ results at the $\mathrm{S}_{1}$ MEP FC- ${ }^{1}\left(\pi, \pi^{*}\right)_{\min }$ structures along the path and the corresponding SOC values are displayed in Figure SI2.

Location of $\mathbf{S T C} \boldsymbol{C}_{1}$ and $\boldsymbol{S T C _ { 2 }}$. For structures $\mathbf{S T C}_{\mathbf{1}}$ and $\mathbf{S T C} \mathbf{C}_{2}$, the proximity of several singlet and triplet states in the region of ${ }^{1}\left(\pi, \pi^{*}\right)_{\min }$ precludes their optimization using the algorithms mentioned above. Therefore, structure $\mathbf{S T C}_{\mathbf{1}}$ (crossing between the ${ }^{1}\left(\pi, \pi^{*}\right.$ ) and ${ }^{3}\left(\mathrm{n}_{\mathrm{O}}, \pi^{*}\right)$ states) was located by following linear displacements (CASPT2 energies, see main text) from ${ }^{3}\left(\mathbf{n}_{\mathbf{O}}, \pi^{*}\right)_{\min }$ along the gradient difference coordinate between the two states at the starting point (CASSCF gradients). Structure $\mathbf{I S C}_{\mathbf{2}}$ (crossing between the ${ }^{1}\left(\pi, \pi^{*}\right)$ and ${ }^{3}\left(\pi, \pi^{*}\right)$ states) was located analogously, starting the displacements from ${ }^{1}\left(\pi, \pi^{*}\right)_{\min }$. The results of the linear displacement calculations are shown in Figures SI3 and SI4, and the structures are given in Table SI2.

Linear interpolation between ${ }^{1}\left(\pi, \pi^{*}\right)_{\min }$ and $\left(\boldsymbol{S}_{1} / \boldsymbol{S}_{0}\right)_{X} . \operatorname{CASSCF}(12,9)$ and the respective CASPT2 reaction paths were built by linearly interpolated internal coordinates (LI-IC) between the structures of interest. ${ }^{2}$ The potential energy curves so built are displayed in Figure SI5. To summarize, a larger barrier height $\left(9.5 \mathrm{kcal} \mathrm{mol}^{-1}\right.$, CASPT2 result) is found along the LI-IC path with respect to the CASSCF-MEP/6-31G(d) result $\left(4.0 \mathrm{kcal} \mathrm{mol}^{-1}\right)$. The top of the barrier coincides with a further point of $\mathrm{T}_{1} / \mathrm{S}_{1}$ degeneracy (labeled $\left(\mathbf{S 1} 1_{1} / \mathbf{T}_{1}\right)_{\mathbf{X}}$ in Figures SI5 and SI6; the geometry is listed in Table SI2, and the structure is shown in Figure SI7, where the natural orbitals involved in the description of the triplet states are also included). Notice the different behavior of the CASSCF and CASPT2 paths in Figure SI5, top and bottom, respectively. Figure SI6 compiles the computed SOCs in this region and the spin-orbit interaction ( $\mathrm{f}_{\mathrm{SO}}$ ) factor according to Turro: ${ }^{6}$

$$
\mathrm{f}_{\mathrm{SO}}=\frac{\left\langle\Psi_{i}\left|\hat{H}_{S O}\right| \Psi_{f}\right\rangle^{2}}{\Delta E^{2}}
$$

The CASPT2 energy differences (in $\mathrm{cm}^{-1}$ ) were employed in the denominator of the $\mathrm{f}_{\mathrm{SO}}$ factor for the states of interest, whereas the numerator just corresponds to the square of the SOC value. A larger $\mathrm{f}_{\mathrm{SO}}$ factor implies a larger observed rate constant $\mathrm{k}_{\mathrm{ISC}}$. From the representation of the $f_{S O}$ factor with respect to the geometry change, the involvement of 
mainly the $S_{1} / T_{1}$ pair in the intersystem crossing mechanism becomes clear. Moreover, ISC is important in large part of this region.

Conformers in the region of ${ }^{3}\left(\pi, \pi^{*}\right)_{\min }$. In this region we have optimized two minima which correspond to different conformers of this species, with the same electronic character. The two conformers differ in approximately $0.5 \mathrm{kcal} \mathrm{mol}^{-1}$. Moreover, in the vicinity of these structures we have optimized two structures of $T_{1} / S_{0}$ crossing for decay of the triplet to the ground state $\left(\left(\mathbf{T}_{\mathbf{1}} / \mathbf{S}_{\mathbf{0}}\right)_{\mathbf{X}}\right.$, Figure $\left.1 \mathrm{~b}\right)$, which are different conformational minima in the (N-1)-dimensional space of $\mathrm{T}_{1} / \mathrm{S}_{0}$ degeneracy. Both structures differ by approximately $2.8 \mathrm{kcal} \mathrm{mol}^{-1}$. The geometries of the two ${ }^{3}\left(\pi, \pi^{*}\right)_{\min }$ conformers are given in Table SI1 and shown in Figure SI1, while the ones for the two $\left(\mathbf{T}_{\mathbf{1}} / \mathbf{S}_{\mathbf{0}}\right)_{\mathbf{X}}$ conformers appear in Table SI2 and Figure SI7. The CASPT2//CASSCF barrier for the decay from $T_{1}$ to $S_{0}$ is estimated from the energy difference between the two lowest conformers and is approximately $4.8 \mathrm{kcal} \mathrm{mol}^{-1}$.

Calculated MEP on the $T_{1}$ and $S_{0}$ surface. The MEP from $\left(\mathbf{S}_{\mathbf{1}} / \mathbf{T}_{\mathbf{1}}\right)_{\mathbf{X}}$ to ${ }^{3}\left(\pi, \pi^{*}\right)_{\min }$ (CASSCF and CASPT2 data) is shown in Figure SI8. The MEP from $\left(\mathbf{T}_{\mathbf{0}} / \mathbf{S}_{\mathbf{1}}\right)_{\mathbf{x}}$ to ${ }^{3}\left(\pi, \pi^{*}\right)_{\min }$ is shown in Figure SI9, together with the corresponding SOCs and $\mathrm{f}_{\mathrm{SO}}$ factors are reported in Figure SI10. The MEP from $\left(\mathbf{T}_{\mathbf{0}} / \mathbf{S}_{1}\right)_{\mathbf{X}}$ to $(\mathbf{g s})_{\min }$ is presented in Figure SI11.

Analysis of the SOC values. In the CASSCF formalism, the components of the SOC vector $\left\langle\mathrm{T}_{1, \mathrm{u}}\left|\hat{\mathrm{H}}_{\mathrm{SO}}\right| \mathrm{S}_{\mathrm{k}}\right\rangle$ are decomposed into sums of the form:

$$
\left\langle\mathrm{T}_{l, u}\left|\hat{\mathrm{H}}_{\mathrm{SO}}\right| \mathrm{S}_{k}\right\rangle=\sum_{p, q}\left\langle\phi_{p}\left|\hat{\mathrm{H}}_{\mathrm{L}, \mathrm{u}}\right| \phi_{q}\right\rangle P_{p q}^{\alpha \beta}
$$

where $\left\langle\phi_{p}\left|\hat{\mathrm{H}}_{\mathrm{L}, \mathrm{u}}\right| \phi_{q}\right\rangle$ are the spin-orbit coupling integrals in the basis of molecular orbitals, and $P_{p q}^{\alpha \beta}$ are the transition spin density matrix elements between configurations of the active space. ${ }^{7,8}$ To rationalize the increased spin-orbit coupling along the MEP for singlet state decay, we have identified the most significant terms of the sum in a basis of localized active-space orbitals. ${ }^{9}$ As one can expect from the Salem-Rowland rules, ${ }^{10}$ the largest integrals are the ones between the in-plane and out-of-plane oxygen active orbitals ( $\mathrm{n}_{\mathrm{O}}$ and $\pi_{\mathrm{O}}$, respectively). The corresponding transition spin density matrix components involve $\left(\pi, \pi^{*}\right)$ and $\left(\mathrm{n}, \pi^{*}\right)$ type configurations and would be approximately 0.0 for "pure" 
${ }^{3}\left(\pi, \pi^{*}\right) /^{1}\left(\pi, \pi^{*}\right)$ or $^{3}\left(\mathrm{n}, \pi^{*}\right) /^{1}\left(\mathrm{n}, \pi^{*}\right)$ transitions, and approximately 1.0 for $^{3}\left(\mathrm{n}, \pi^{*}\right) /^{1}\left(\pi, \pi^{*}\right)$ or ${ }^{3}\left(\pi, \pi^{*}\right) /^{1}\left(\mathrm{n}, \pi^{*}\right)$ ones. In the region of $\mathbf{S T C}_{3}$, the terms are high (approximately 0.5 ) because the wave functions of the singlet and triplet states are multireferential due to the vibronic coupling between the $\left(\pi, \pi^{*}\right)$ and $\left(n, \pi^{*}\right)$ states. ${ }^{3}$ Thus the calculated SOC value is the result of the interaction between $\left(\pi, \pi^{*}\right)$ and $\left(\mathrm{n}, \pi^{*}\right)$ configurations.

S5. Calculation of the intersystem crossing probability $\left(P^{I S C}\right)$.

The probability for ISC at a crossing, based on a Landau-Zener-type model, ${ }^{11}$ is given by:

$$
\begin{aligned}
& P^{I S C}=1-P^{L Z} \\
& P^{L Z}=\exp \left(-\frac{\pi}{4} \xi\right) \\
& \xi=\frac{8 H_{S O C}^{2}}{\hbar \mathbf{g d} \overrightarrow{\mathbf{v}} N_{A v}}
\end{aligned}
$$

Here $P^{I S C}$ is calculated at $\mathbf{S T C}_{\mathbf{3}}$. The calculated SOC is $27.1 \mathrm{~cm}^{-1}$, ie $1.235 \times 10^{-4}$ hartree. $\overrightarrow{\text { gd }}$ is the gradient difference vector between both states. For the calculation of $\overrightarrow{\mathbf{v}}$, it is assumed that the excess excitation energy $\left(\mathrm{S}_{1}\right.$ energy at $(\mathbf{g s})_{\min }-\mathrm{S}_{1}$ energy at $\left.\mathbf{I S C}_{\mathbf{2}}\right)$ is converted into kinetic energy and the corresponding velocity vector is parallel to the gradient difference vector. This is the most unfavorable case for ISC, so the calculated value gives a lower limit for $P^{I S C}$. Using this approximation, the $\overrightarrow{\text { gd }} \overrightarrow{\mathbf{v}}$ vector product is equal to the product of the norms of the two vectors. The calculated values for $\overrightarrow{g d}$ and $\overrightarrow{\mathbf{v}}$ are 0.07006 hartree bohr $^{-1}$ and 0.0013908 bohr per atomic time unit, respectively. These values give $P^{I S C}=1 * 10^{-3}$.

\section{References}

(1) Ismail, N.; Blancafort, L.; Olivucci, M.; Kohler, B.; Robb, M. A. J. Am. Chem. Soc. 2002, 124, 6818-6819.

(2) Merchán, M.; Serrano-Andrés, L. J. Am. Chem. Soc. 2003, 125, 8108-8109.

(3) Blancafort, L.; Robb, M. A. J. Am. Chem. Soc. 2004, in press.

(4) Andersson, K.; Barysz, M.; Bernhardsson, A.; Blomberg, M. R. A.; Carissan, Y.; Cooper, D. L.; Cossi, M.; Fülscher, M. P.; Gagliardi, L.; Graaf, C. d.; Hagberg, G.; Hess, B. A.; Karlström, G.; Lindh, R.; Malmqvist, P.-Å.; Nakajima, T.; 
Neogrády, P.; Olsen, J.; Raab, J.; Roos, B. O.; Ryde, U.; Schimmelpfennig, B.; Schütz, M.; Seijo, L.; Serrano-Andrés, L.; Siegbahn, P. E. M.; Stålring, J.; Thorsteinsson, T.; Veryazov, V.; Widmark, P. O.; MOLCAS Version 6.0 ed.; Department of Theoretical Chemistry, Chemical Centre, University of Lund, P.O.B. 124, S-22100 Lund: Sweden, 2004.

(5) Frisch, M. J.; Trucks, G. W.; Schlegel, H. B.; Scuseria, G. E.; Robb, M. A.; Cheeseman, J. R.; J. A. Montgomery, J.; Vreven, T.; Kudin, K. N.; Burant, J. C.; Millam, J. M.; Iyengar, S. S.; Tomasi, J.; Barone, V.; Mennucci, B.; Cossi, M.; Scalmani, G.; Rega, N.; Petersson, G. A.; Nakatsuji, H.; Hada, M.; Ehara, M.; Toyota, K.; Fukuda, R.; Hasegawa, J.; Ishida, M.; Nakajima, T.; Honda, Y.; Kitao, O.; Nakai, H.; Klene, M.; Li, X.; Knox, J. E.; Hratchian, H. P.; Cross, J. B.; Adamo, C.; Jaramillo, J.; Gomperts, R.; Stratmann, R. E.; Yazyev, O.; Austin, A. J.; Cammi, R.; Pomelli, C.; Ochterski, J. W.; Ayala, P. Y.; Morokuma, K.; Voth, G. A.; Salvador, P.; Dannenberg, J. J.; Zakrzewski, V. G.; Dapprich, S.; Daniels, A. D.; Strain, M. C.; Farkas, O.; Malick, D. K.; Rabuck, A. D.; Raghavachari, K.; Foresman, J. B.; Ortiz, J. V.; Cui, Q.; Baboul, A. G.; Clifford, S.; Cioslowski, J.; Stefanov, B. B.; Liu, G.; Liashenko, A.; Piskorz, P.; Komaromi, I.; Martin, R. L.; Fox, D. J.; Keith, T.; Al-Laham, M. A.; Peng, C. Y.; Nanayakkara, A.; Challacombe, M.; Gill, P. M. W.; Johnson, B.; Chen, W.; Wong, M. W.; Gonzalez, C.; Pople, J. A.; Gaussian03 Revision B.02 ed.; Gaussian, Inc.: Pittsburgh, PA, 2003.

(6) Turro, N. J. Modern Molecular Photochemistry; University Science Books: Sausalito, CA, 1991.

(7) Wilsey, S. Ph. D. Thesis; University of London, 1996.

(8) Malmqvist, P. A.; Roos, B. O.; Schimmelpfennig, B. Chem.

Phys. Lett. 2002, 357, 230-240.

(9) Boys, S. F. Reviews of Modern Physics 1960, 32, 296-299.

(10) Salem, L.; Rowland, C. Angewandte Chemie-International

Edition 1972, 11, 92-\&.

(11) Manaa, M. R.; Yarkony, D. R. J. Chem. Phys. 1991, 95, 1808-

1816. 
Table SI1. Cartesian coordinates $(\AA)$ for the relevant triplet state structures of cytosine.

$\begin{array}{lll}\mathrm{x} & \mathrm{y} & \mathrm{z}\end{array}$

${ }^{3}\left(\pi, \pi^{*}\right)_{\min }$ low-energy conformer.

$\begin{array}{llll}\mathrm{N} & -0.197510 & 1.318603 & -0.071858\end{array}$

$\begin{array}{llll}\mathrm{C} & -0.022707 & 2.507156 & -0.758810\end{array}$

$\begin{array}{llll}\mathrm{N} & 0.154136 & 2.469408 & -2.150701\end{array}$

$\begin{array}{llll}\text { C } & 0.026233 & 1.346604 & -2.784210\end{array}$

$\begin{array}{llll}\text { C } & -0.215212 & 0.099431 & -2.120749\end{array}$

$\begin{array}{llll}C & -0.032405 & 0.059992 & -0.644636\end{array}$

$\begin{array}{llll}\mathrm{O} & -0.024013 & 3.554533 & -0.172062\end{array}$

$\begin{array}{llll}\mathrm{N} & 0.020882 & 1.368874 & -4.167793\end{array}$

H $\quad 0.284621 \quad 2.255026 \quad-4.543594$

$\mathrm{H} \quad-0.658986-0.722204 \quad-2.648391$

$\mathrm{H} \quad 0.747449 \quad-0.563144 \quad-0.229175$

$\mathrm{H} \quad 0.484160 \quad 0.607493 \quad-4.614322$

$\mathrm{H} \quad-0.166654 \quad 1.4141120 .919090$

${ }^{3}\left(\pi, \pi^{*}\right)_{\min }$ high-energy conformer.

$\begin{array}{lrrr}\mathrm{N} & -0.294538 & 1.549582 & 0.005794 \\ \mathrm{C} & 0.888147 & 0.846772 & 0.003540 \\ \mathrm{~N} & 0.862070 & -0.548868 & -0.010401 \\ \mathrm{C} & -0.301850 & -1.171674 & -0.029069 \\ \mathrm{C} & -1.530929 & -0.505750 & 0.037224 \\ \mathrm{C} & -1.554525 & 0.986546 & 0.157609 \\ \mathrm{O} & 1.943300 & 1.431509 & -0.004386 \\ \mathrm{~N} & -0.262606 & -2.556481 & -0.143060 \\ \mathrm{H} & 0.648147 & -2.925726 & 0.022495 \\ \mathrm{H} & -2.454888 & -1.050157 & 0.030046 \\ \mathrm{H} & -2.356195 & 1.524200 & -0.321487 \\ \mathrm{H} & -0.968538 & -3.045642 & 0.360435 \\ \mathrm{H} & -0.189821 & 2.538413 & 0.013990\end{array}$


Table SI1 (cont). Cartesian coordinates $(\AA)$ for the relevant triplet state structures of cytosine.

\begin{tabular}{lrrc} 
& \multicolumn{1}{c}{$\mathrm{x}$} & $\mathrm{y}$ & $\mathrm{Z}$ \\
${ }^{\mathbf{3}}\left(\mathbf{n}_{\mathbf{O}}, \pi^{*}\right)_{\min }$ & & & \\
$\mathrm{N}$ & -0.285075 & 1.559514 & 0.117561 \\
$\mathrm{C}$ & 0.828839 & 0.765162 & 0.021491 \\
$\mathrm{~N}$ & 0.875451 & -0.496001 & -0.014373 \\
$\mathrm{C}$ & -0.344812 & -1.175791 & -0.005454 \\
$\mathrm{C}$ & -1.542240 & -0.475356 & -0.024711 \\
$\mathrm{C}$ & -1.535185 & 0.913080 & -0.005025 \\
$\mathrm{O}$ & 1.975912 & 1.476237 & 0.020478 \\
$\mathrm{~N}$ & -0.232877 & -2.562039 & -0.135359 \\
$\mathrm{H}$ & 0.636912 & -2.898700 & 0.218131 \\
$\mathrm{H}$ & -2.480427 & -0.997523 & -0.065015 \\
$\mathrm{H}$ & -2.404524 & 1.530571 & 0.065666 \\
$\mathrm{H}$ & -0.997625 & -3.066739 & 0.256886 \\
$\mathrm{H}$ & -0.201558 & 2.501873 & -0.190553 \\
& & & \\
$\mathbf{3}\left(\mathbf{n}_{\mathbf{N}}, \pi^{*}\right)_{\min }$ & & & \\
& & & \\
$\mathrm{N}$ & -0.013836 & -0.281374 & 1.547731 \\
$\mathrm{C}$ & -0.003306 & 0.940774 & 0.912509 \\
$\mathrm{~N}$ & -0.000100 & 0.766947 & -0.444997 \\
$\mathrm{C}$ & 0.035444 & -0.381111 & -1.235593 \\
$\mathrm{C}$ & 0.020711 & -1.557814 & -0.507585 \\
$\mathrm{C}$ & -0.014867 & -1.516528 & 0.883388 \\
$\mathrm{O}$ & -0.001547 & 1.994860 & 1.477994 \\
$\mathrm{~N}$ & -0.059441 & -0.193187 & -2.616381 \\
$\mathrm{H}$ & 0.656008 & 0.409667 & -2.969327 \\
$\mathrm{H}$ & 0.029182 & -2.503563 & -1.016089 \\
$\mathrm{H}$ & -0.018853 & -2.387334 & 1.502900 \\
$\mathrm{H}$ & -0.039557 & -1.058409 & -3.110715 \\
$\mathrm{H}$ & -0.034960 & -0.240877 & 2.539833
\end{tabular}


Table SI2. Cartesian coordinates $(\AA)$ for the structures of singlet/triplet degeneracy.

X $\mathrm{y}$

Z

STC $_{1}$

$\begin{array}{lrrr}\mathrm{N} & 0.122790 & 1.370216 & -0.033079 \\ \mathrm{C} & 0.044474 & 2.471499 & -0.875588 \\ \mathrm{~N} & 0.050618 & 2.519727 & -2.109125 \\ \mathrm{C} & 0.075069 & 1.313608 & -2.794984 \\ \mathrm{C} & 0.036093 & 0.087657 & -2.062778 \\ \mathrm{C} & -0.022858 & 0.103869 & -0.735063 \\ \mathrm{O} & 0.020965 & 3.630092 & -0.149406 \\ \mathrm{~N} & 0.208301 & 1.401227 & -4.190305 \\ \mathrm{H} & -0.102179 & 2.294315 & -4.509032 \\ \mathrm{H} & 0.044991 & -0.841021 & -2.612061 \\ \mathrm{H} & 0.048825 & -0.757257 & -0.084963 \\ \mathrm{H} & -0.253760 & 0.671304 & -4.688012 \\ \mathrm{H} & -0.273337 & 1.450663 & 0.857205\end{array}$

$\mathrm{STC}_{2}$

$\begin{array}{lrrr}\mathrm{N} & -0.021150 & 1.362218 & -0.085319 \\ \mathrm{C} & 0.014650 & 2.477141 & -0.864866 \\ \mathrm{~N} & 0.058743 & 2.532645 & -2.123744 \\ \mathrm{C} & 0.067266 & 1.291877 & -2.792740 \\ \mathrm{C} & 0.006326 & 0.102331 & -2.106203 \\ \mathrm{C} & -0.102643 & 0.102162 & -0.706520 \\ \mathrm{O} & 0.009284 & 3.598245 & -0.112648 \\ \mathrm{~N} & 0.218863 & 1.409583 & -4.176699 \\ \mathrm{H} & -0.128578 & 2.282519 & -4.515000 \\ \mathrm{H} & 0.025597 & -0.833335 & -2.633213 \\ \mathrm{H} & 0.081311 & -0.752380 & -0.089240 \\ \mathrm{H} & -0.163351 & 0.646319 & -4.694787 \\ \mathrm{H} & -0.100386 & 1.452054 & 0.900110\end{array}$


Table SI2. Cartesian coordinates $(\AA)$ for the structures of singlet/triplet degeneracy.

$\mathrm{X}$

$\mathrm{STC}_{3}$

$\begin{array}{lrrr}\mathrm{N} & -0.024301 & 1.364092 & -0.098057 \\ \mathrm{C} & 0.015805 & 2.477211 & -0.875898 \\ \mathrm{~N} & 0.054432 & 2.533510 & -2.128645 \\ \mathrm{C} & 0.072521 & 1.282287 & -2.793564 \\ \mathrm{C} & 0.010768 & 0.103892 & -2.124003 \\ \mathrm{C} & -0.103685 & 0.116660 & -0.684885 \\ \mathrm{O} & 0.010409 & 3.592030 & -0.094438 \\ \mathrm{~N} & 0.216510 & 1.408089 & -4.173883 \\ \mathrm{H} & -0.123763 & 2.286308 & -4.505250 \\ \mathrm{H} & 0.035221 & -0.836613 & -2.636988 \\ \mathrm{H} & 0.065549 & -0.737160 & -0.061450 \\ \mathrm{H} & -0.162138 & 0.649615 & -4.699911 \\ \mathrm{H} & -0.067338 & 1.475980 & 0.889784\end{array}$

$\left(\mathbf{S}_{\mathbf{1}} / \mathbf{T}_{\mathbf{1}}\right)_{\mathbf{X}}$ from LI-IC 
Table SI2. Cartesian coordinates $(\AA)$ for the structures of singlet/triplet degeneracy.

$\mathrm{X}$

$\mathrm{y}$

Z

$\left(\mathbf{T}_{\mathbf{1}} / \mathbf{S}_{\mathbf{0}}\right)_{\mathbf{X}}$ low-energy conformer

$\begin{array}{cccc}\mathrm{N} & -0.276338 & 1.241327 & -0.088207 \\ \mathrm{C} & -0.107299 & 2.457091 & -0.753487 \\ \mathrm{~N} & 0.157679 & 2.436770 & -2.137625 \\ \mathrm{C} & 0.063031 & 1.337980 & -2.790218 \\ \mathrm{C} & -0.260664 & 0.060993 & -2.122484 \\ \mathrm{C} & 0.139273 & 0.049769 & -0.701339 \\ \mathrm{O} & -0.189006 & 3.492722 & -0.159253 \\ \mathrm{~N} & 0.156283 & 1.343443 & -4.156370 \\ \mathrm{H} & 0.474510 & 2.202961 & -4.550116 \\ \mathrm{H} & -1.182815 & -0.412907 & -2.416296 \\ \mathrm{H} & 1.107122 & -0.346967 & -0.426985 \\ \mathrm{H} & 0.527704 & 0.526645 & -4.587773 \\ \mathrm{H} & -0.209484 & 1.326057 & 0.902943\end{array}$

$\left(\mathbf{T}_{\mathbf{1}} / \mathbf{S}_{\mathbf{0}}\right)_{\mathbf{X}}$ high-energy conformer

$\begin{array}{lrrr}\mathrm{N} & -0.372808 & 1.521542 & -0.074371 \\ \mathrm{C} & 0.851836 & 0.859304 & -0.132167 \\ \mathrm{~N} & 0.867787 & -0.524512 & 0.123924 \\ \mathrm{C} & -0.231780 & -1.180060 & 0.077498 \\ \mathrm{C} & -1.520898 & -0.482079 & -0.187416 \\ \mathrm{C} & -1.468595 & 0.863324 & 0.474531 \\ \mathrm{O} & 1.855902 & 1.458154 & -0.395473 \\ \mathrm{~N} & -0.233365 & -2.537787 & 0.179944 \\ \mathrm{H} & 0.628800 & -2.949262 & 0.460130 \\ \mathrm{H} & -1.792675 & -0.488675 & -1.238293 \\ \mathrm{H} & -2.343241 & 1.444582 & 0.691469 \\ \mathrm{H} & -1.057627 & -2.975460 & 0.519910 \\ \mathrm{H} & -0.312247 & 2.514421 & -0.113172\end{array}$



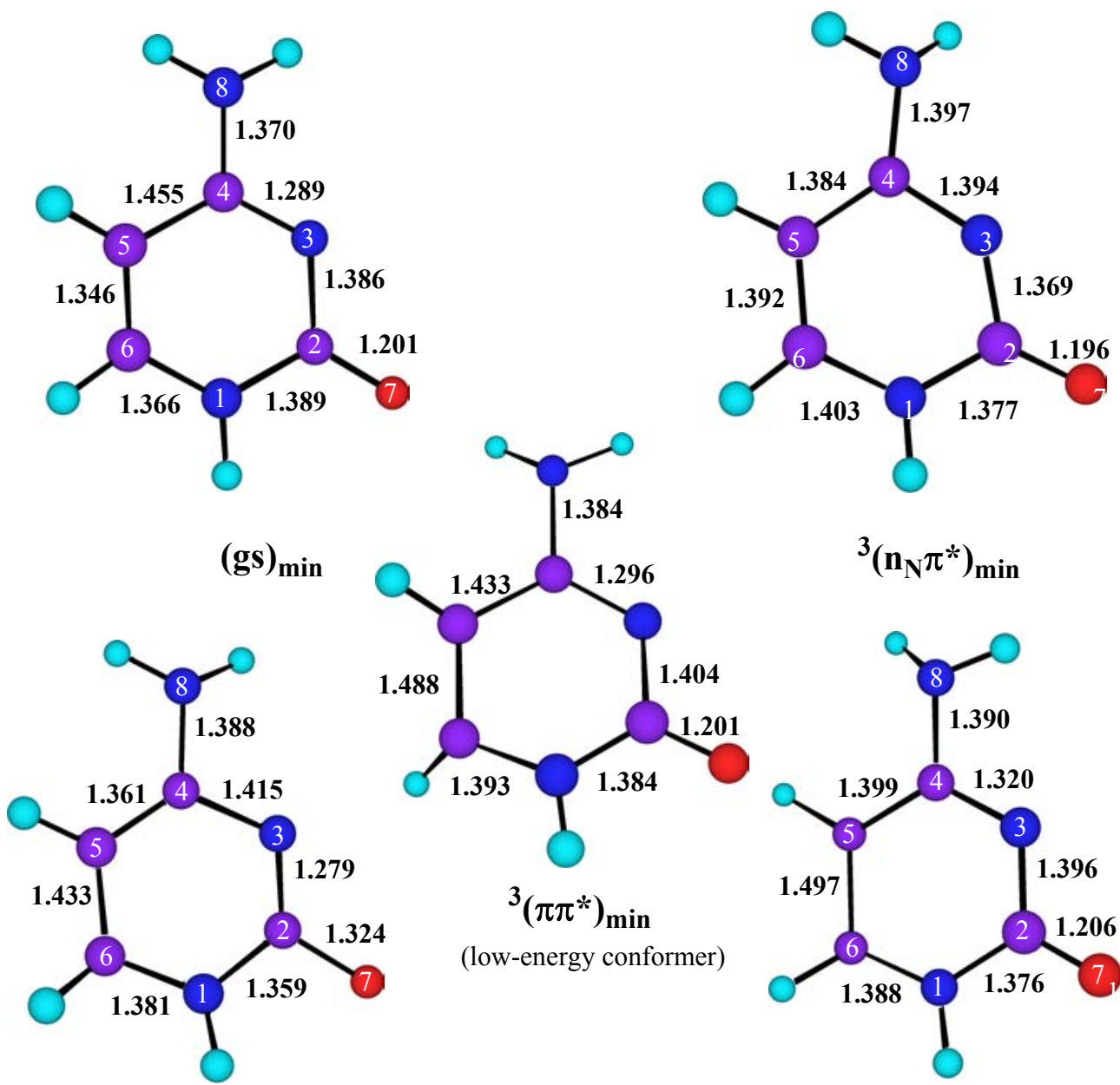

$$
{ }^{1}\left(\pi \pi^{*}\right)_{\min }
$$
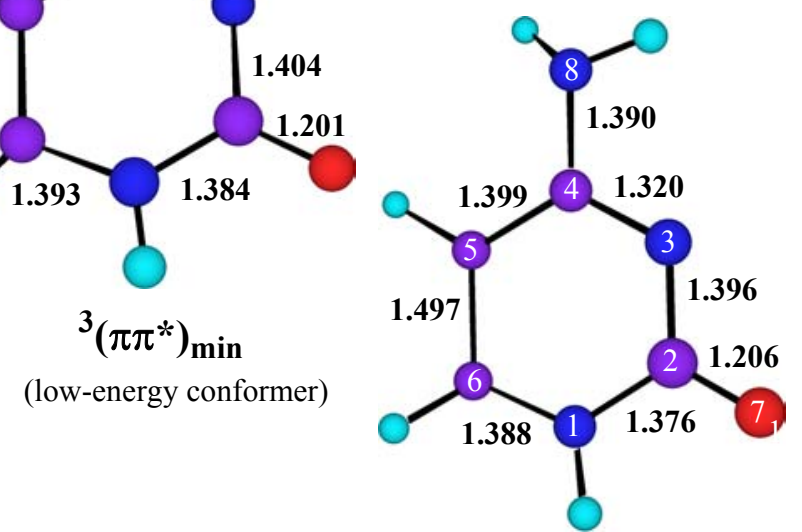

$$
{ }^{3}\left(\pi \pi^{*}\right)_{\text {min }}
$$

(high-energy conformer)

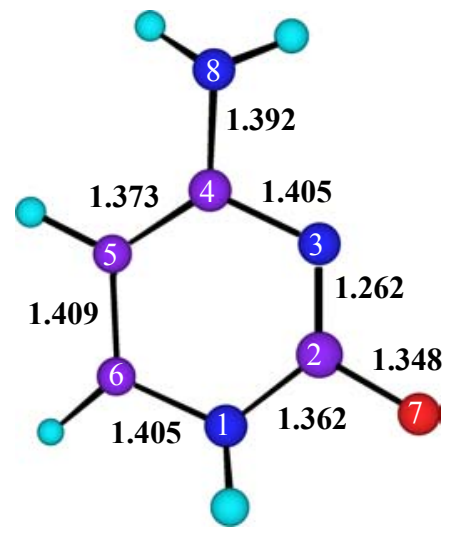

$$
{ }^{1}\left(n_{0} \pi^{*}\right)_{\min }
$$

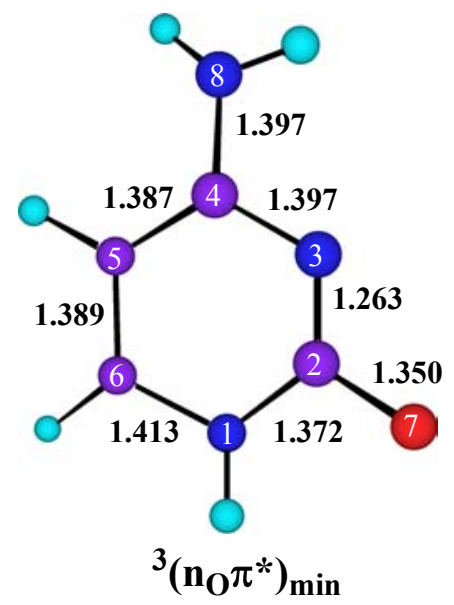

Figure SI1. Optimized CASSCF/6-31G(d,p) structures for the triplet excited states. For the sake of comparison, the equilibrium geometries for the ground and valence singlet states (taken from the Supporting Information of Ref. 2) are also shown. 

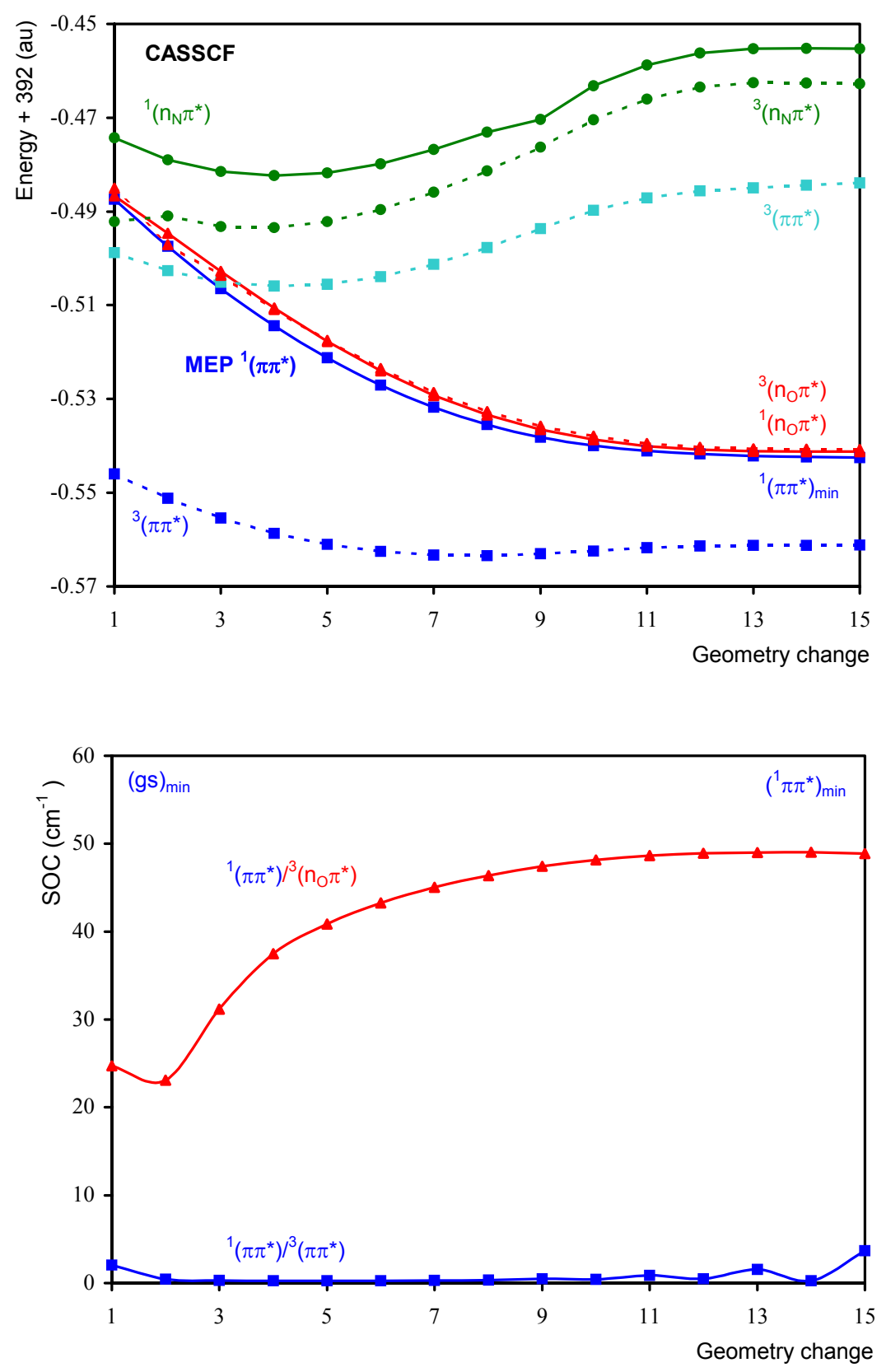

Figure SI2. CASSCF(12,9)/6-31G(d,p) results for the low-lying excited states computed along the $\mathrm{S}_{1}$ MEP FC- ${ }^{1}\left(\pi, \pi^{*}\right)_{\min }$ path and the corresponding SOC. 


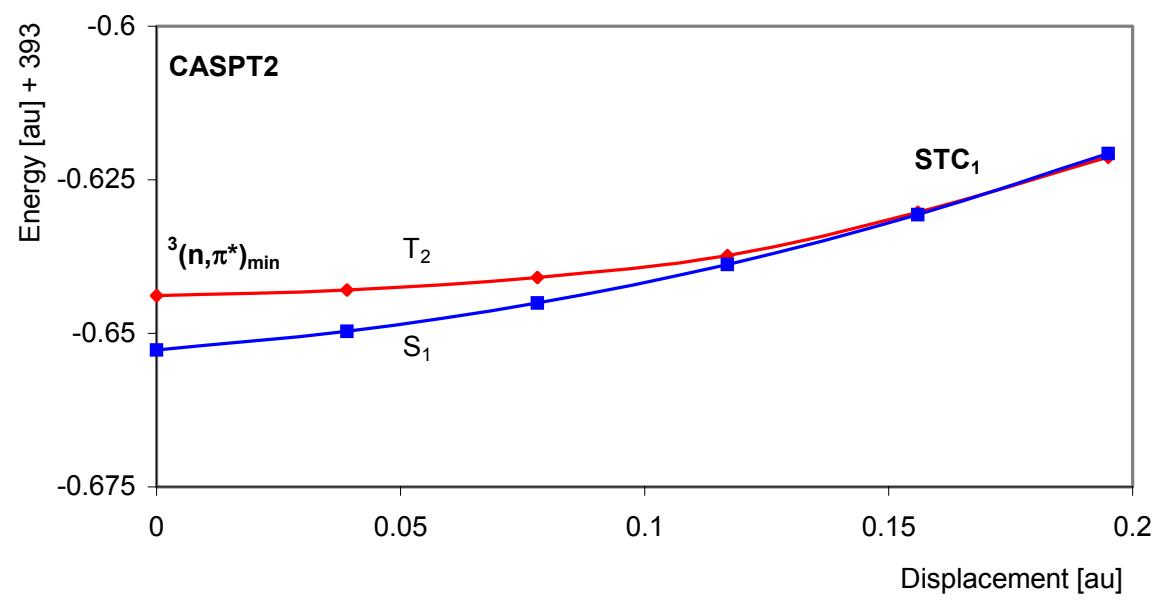

Figure SI3. Linear coordinate for location of $\mathbf{S T C}_{1}$; starting point: ${ }^{3}\left(\mathbf{n}_{\mathbf{0}}, \pi^{*}\right)_{\min }$, direction: gradient difference between $T_{2}$ and $S_{1}$.

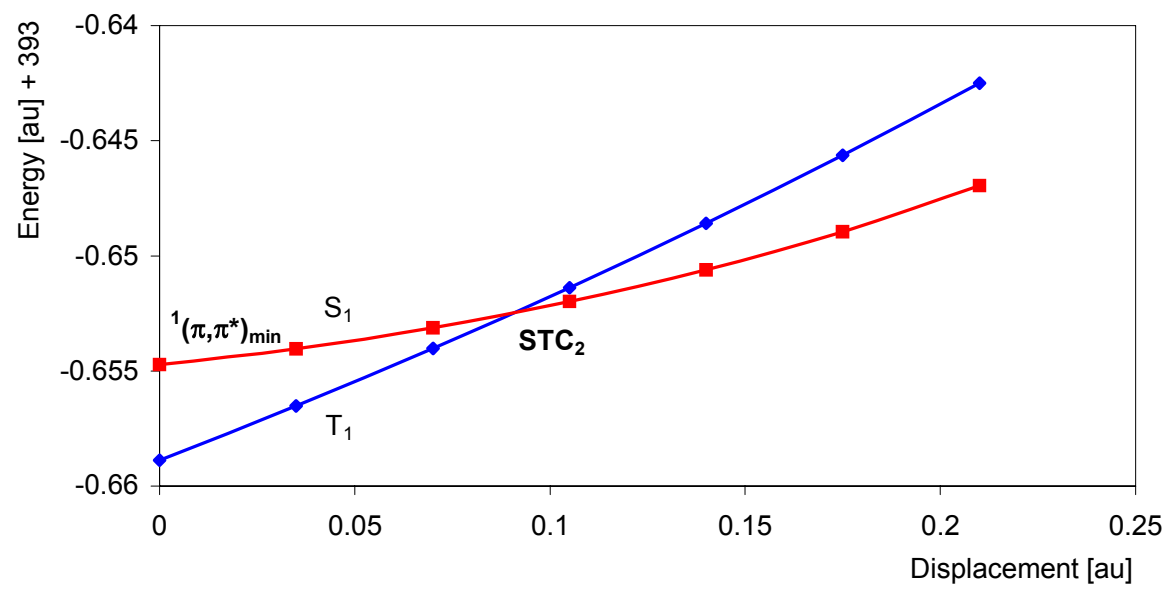

Figure SI4. Linear coordinate for location of $\mathbf{S T C}_{2}$; starting point: ${ }^{1}\left(\pi, \pi^{*}\right)_{\min }$, direction: gradient difference between $T_{1}$ and $S_{1}$. 

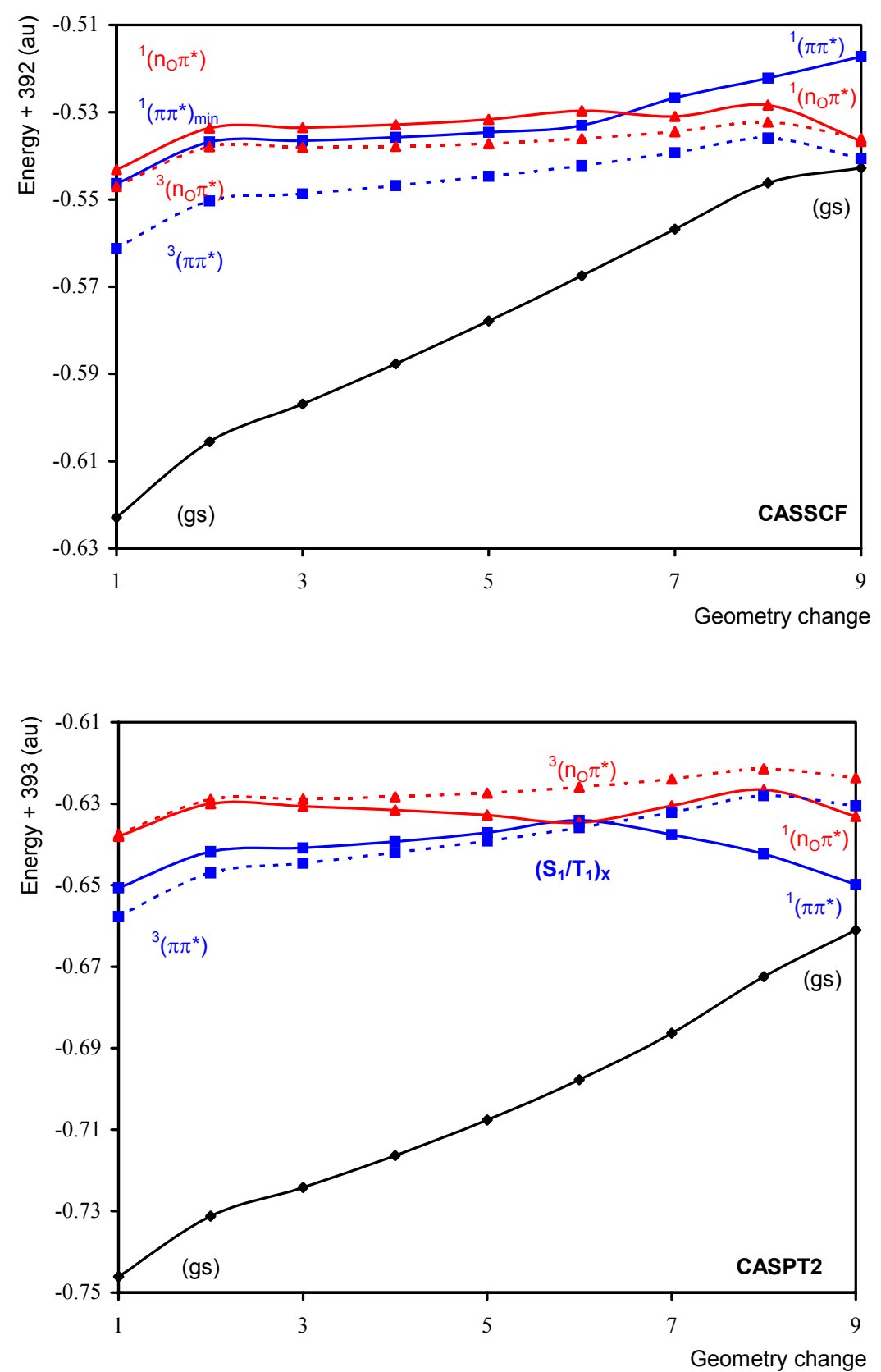

Figure SI5. CASSCF $(12,9) / 6-31 G(d, p)$ results for the region ${ }^{1}\left(\pi, \pi^{*}\right)_{\min }-\left(\mathbf{S}_{\mathbf{1}} / \mathbf{S}_{\mathbf{0}}\right)_{\mathbf{X}}$ built by using LI-IC. 

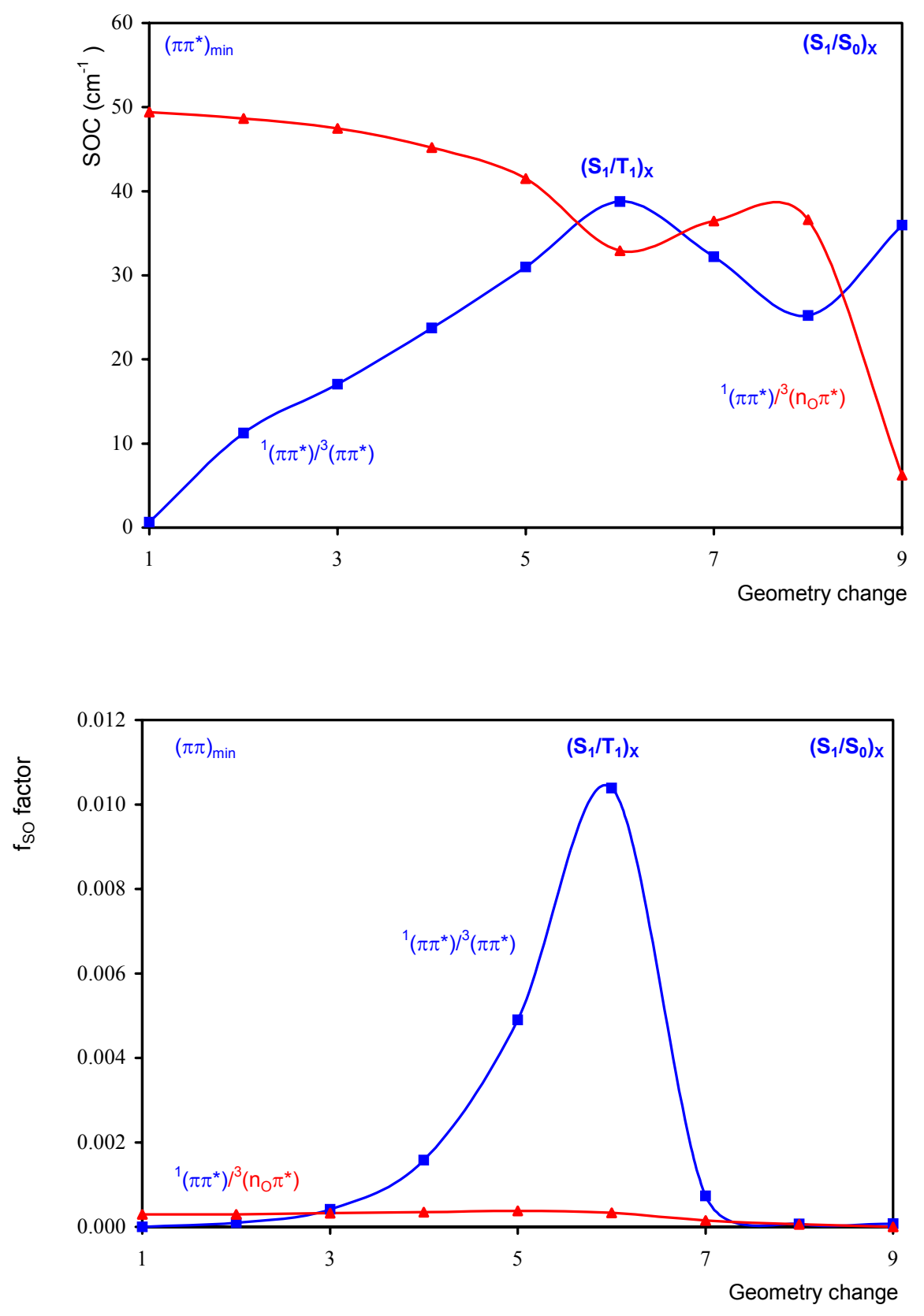

Figure SI6. Computed SOCs (top) and evolution of the $\mathrm{f}_{\mathrm{SO}}$ factor (bottom) for the region ${ }^{1}\left(\pi, \pi^{*}\right)_{\min }-\left(\mathbf{S}_{1} / \mathbf{S}_{0}\right)_{\mathbf{X}}$ built by using LI-IC. 


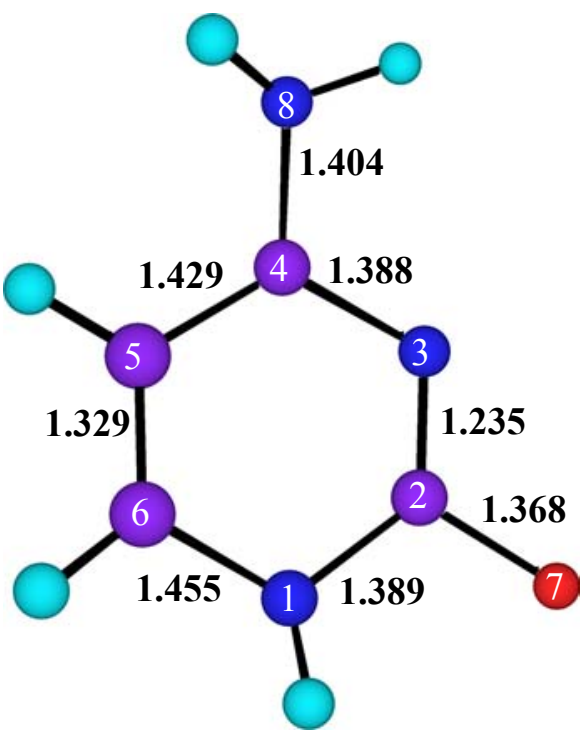

$\mathrm{STC}_{1}$

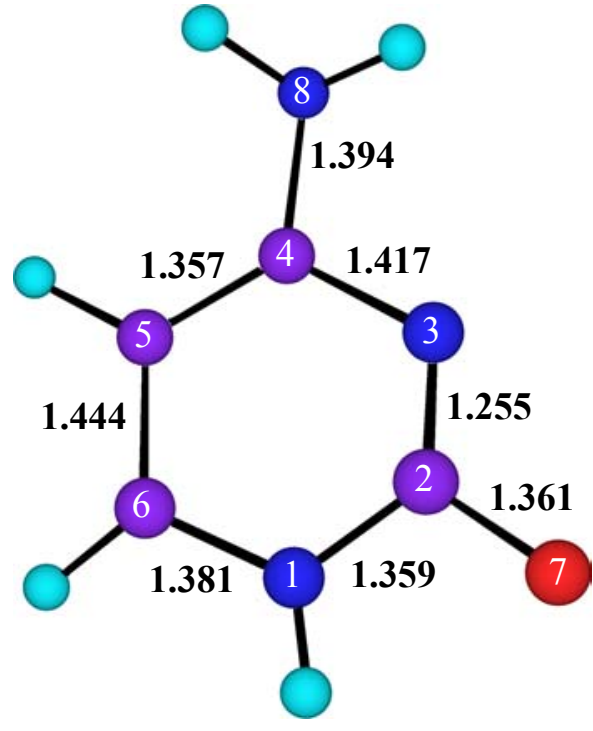

$\mathrm{STC}_{3}$

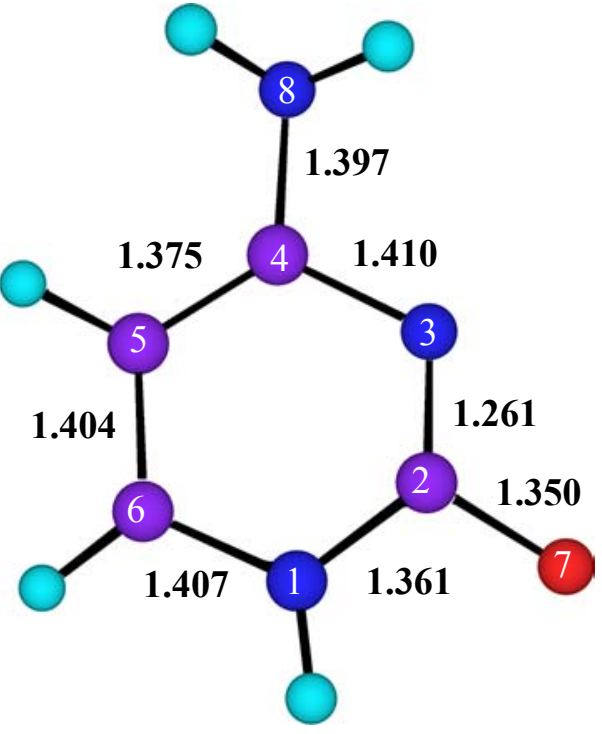

$\mathrm{STC}_{2}$

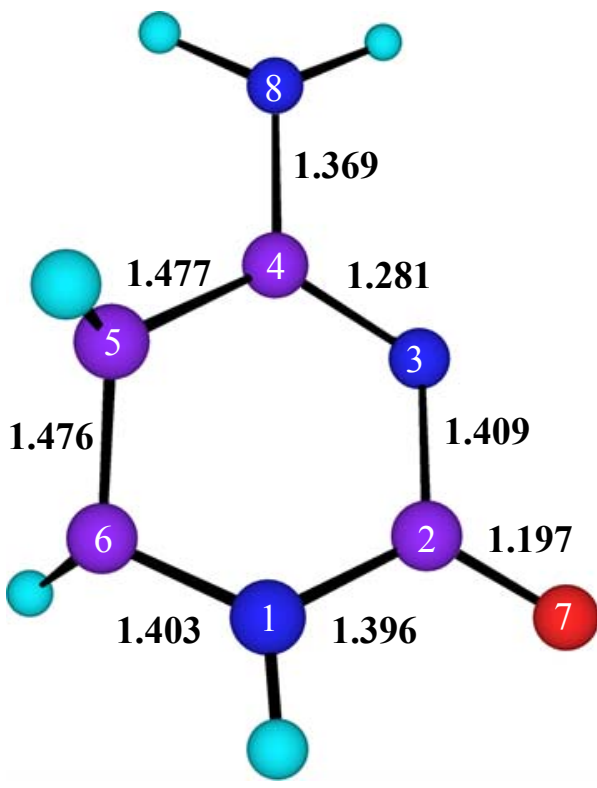

$\left(\mathbf{T}_{1} / \mathbf{S}_{0}\right)_{X}$

(low-energy conformer)

Figure SI7. Structural information on $\mathbf{S T C}_{1}, \mathbf{S T C}_{2}, \mathbf{S T C}_{3},\left(\mathbf{S}_{1} / \mathbf{T}_{1}\right)_{\mathbf{X}}$ and $\left(\mathbf{T}_{1} / \mathbf{S}_{\mathbf{0}}\right)_{\mathbf{X}}($ lowand high-energy conformers) and main natural MOs involved in the description of the triplet state. 


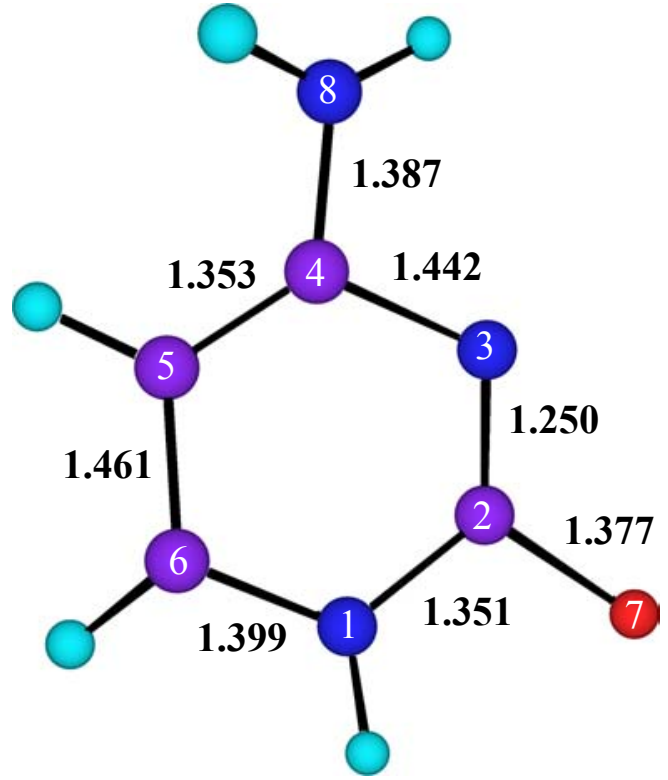

$\left(\mathbf{S}_{1} / \mathbf{T}_{1}\right)_{\mathbf{X}}$

from LI-IC

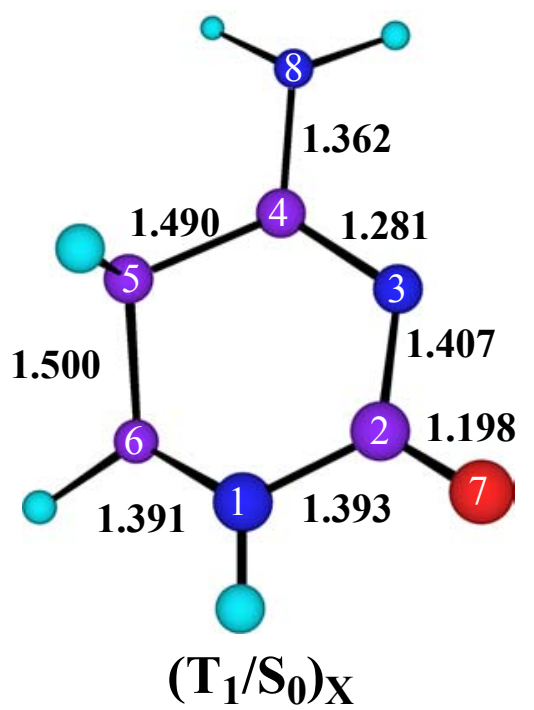

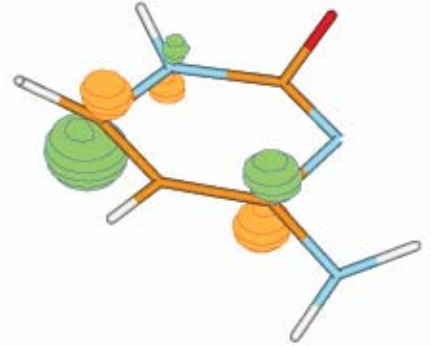

(high-energy conformer)
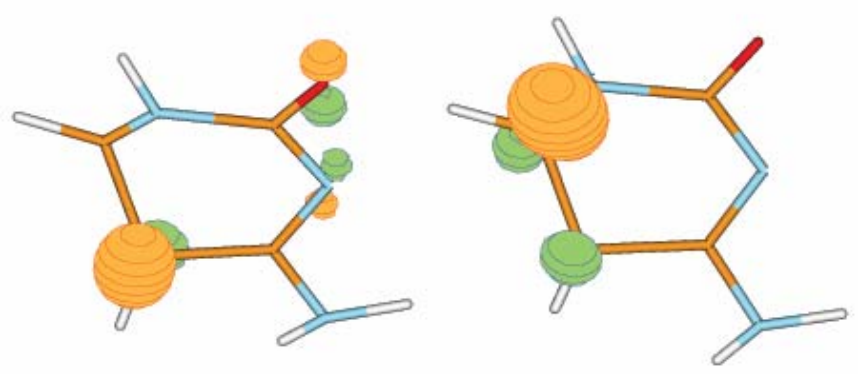

Figure SI7 (cont.). Structural information on $\mathbf{S T C}_{\mathbf{1}}, \mathbf{S T C}_{2}, \mathrm{STC}_{\mathbf{3}},\left(\mathbf{S}_{\mathbf{1}} / \mathbf{T}_{\mathbf{1}}\right)_{\mathrm{X}}$ and $\left(\mathbf{T}_{\mathbf{1}} / \mathbf{S}_{\mathbf{0}}\right)_{\mathrm{X}}$ (low- and high-energy conformers) and main natural MOs involved in the description of the triplet state. 

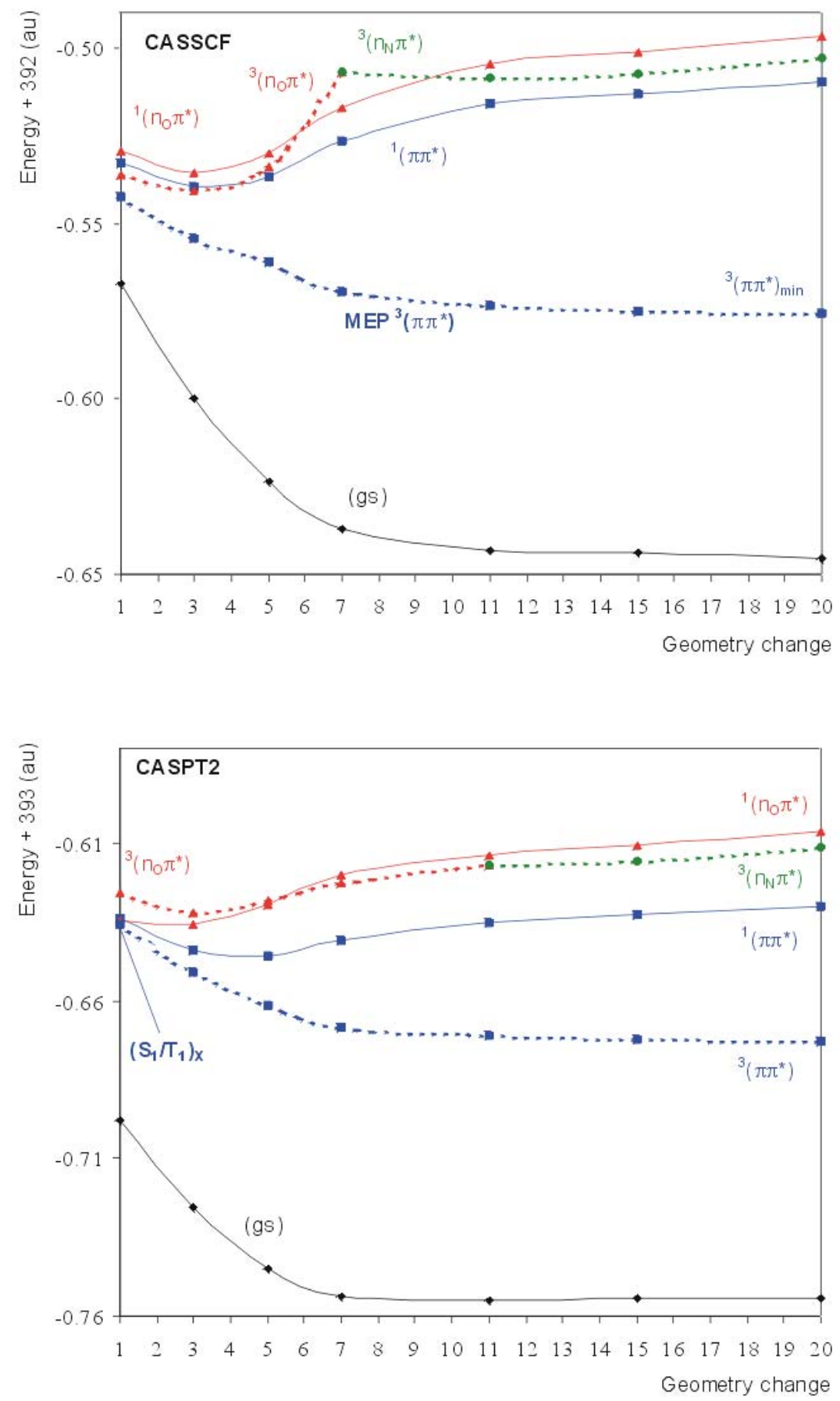

Figure SI8. Along the $\mathrm{T}_{1} \operatorname{CASSCF}(8,7) \operatorname{MEP}$ from $\left(\mathbf{S}_{1} / \mathbf{T}_{1}\right)_{\mathbf{x}}$ leading to ${ }^{3}\left(\pi, \pi^{*}\right)_{\min }$. CASSCF $(12,9)$ results (top) and the corresponding CASPT2 findings (bottom). State average CASSCF $(12,9)$ of three roots for the singlet states and four roots for the triplet states. 

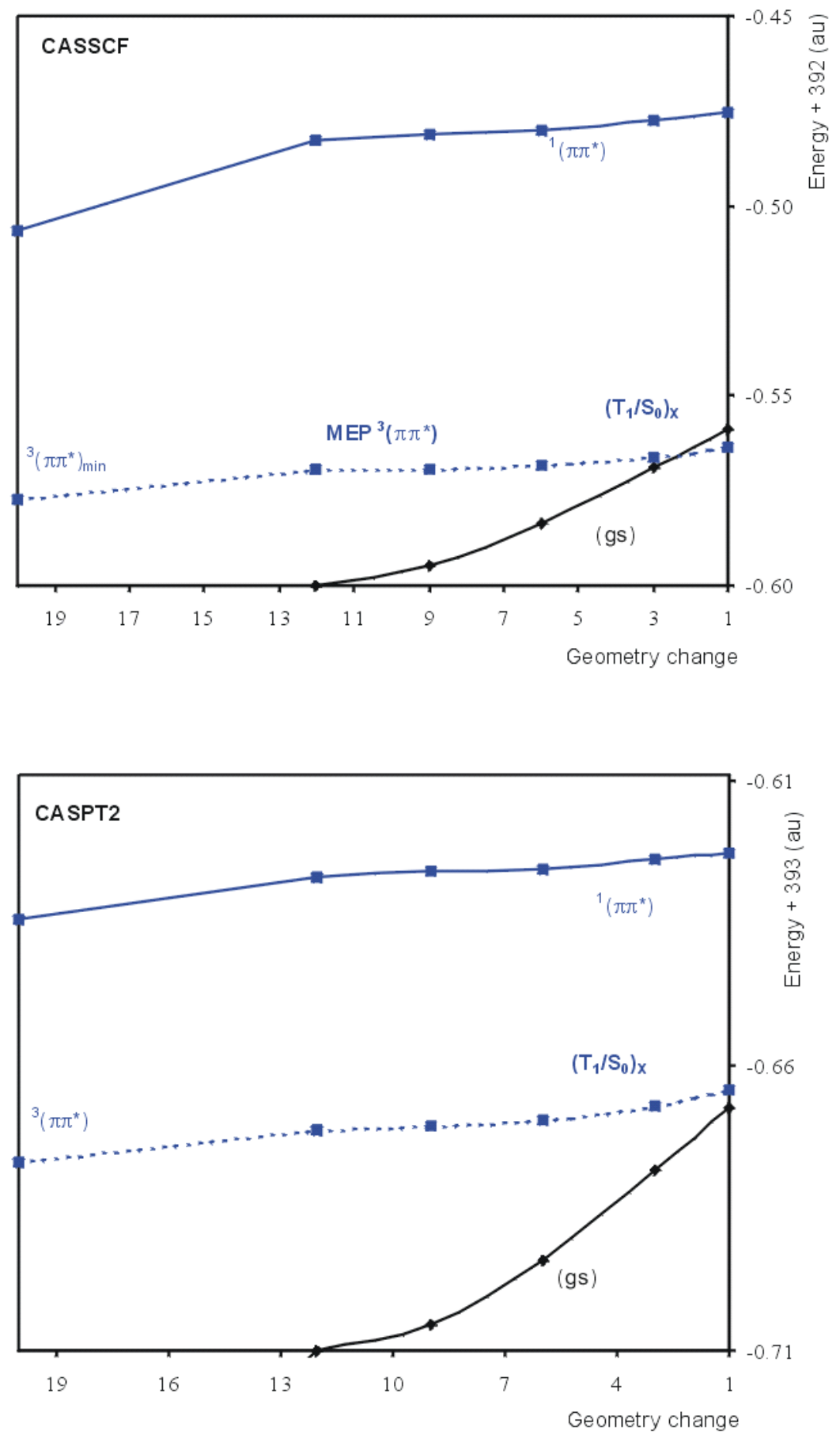

Figure SI9. Along the $\mathrm{T}_{1}$ CASSCF MEP $\left(\mathbf{T}_{1} / \mathbf{S}_{\mathbf{0}}\right)_{\mathbf{X}^{-}}{ }^{3}\left(\pi \pi^{*}\right)_{\min }$ 

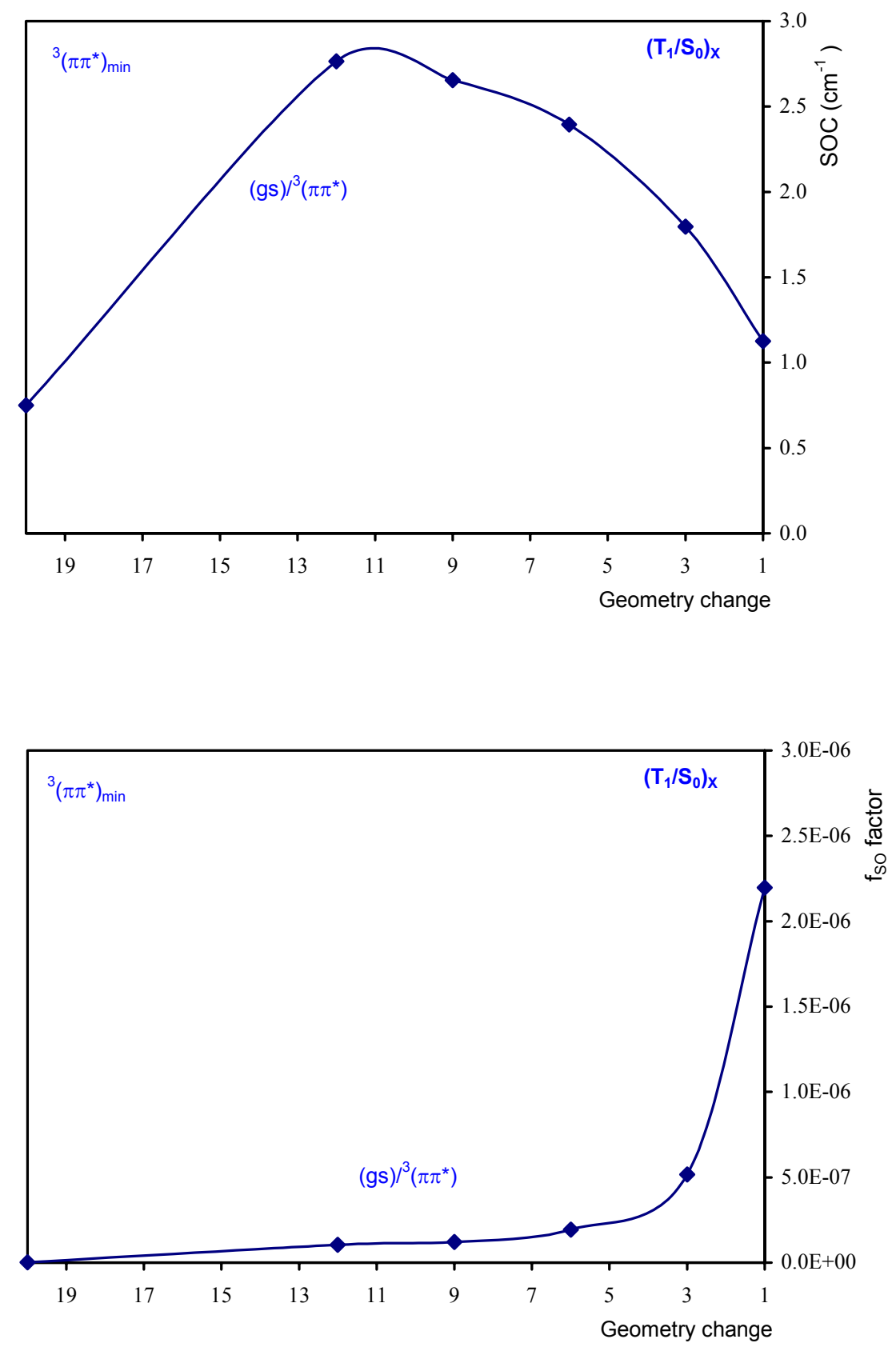

Figure SI10. Computed SOCs and $f_{S O}$ factor along the $T_{1}$ CASSCF MEP $\left(\mathbf{T}_{\mathbf{1}} / \mathbf{S}_{\mathbf{0}}\right)_{\mathbf{X}^{-}}$ ${ }^{3}\left(\pi \pi^{*}\right)_{\min }$ 


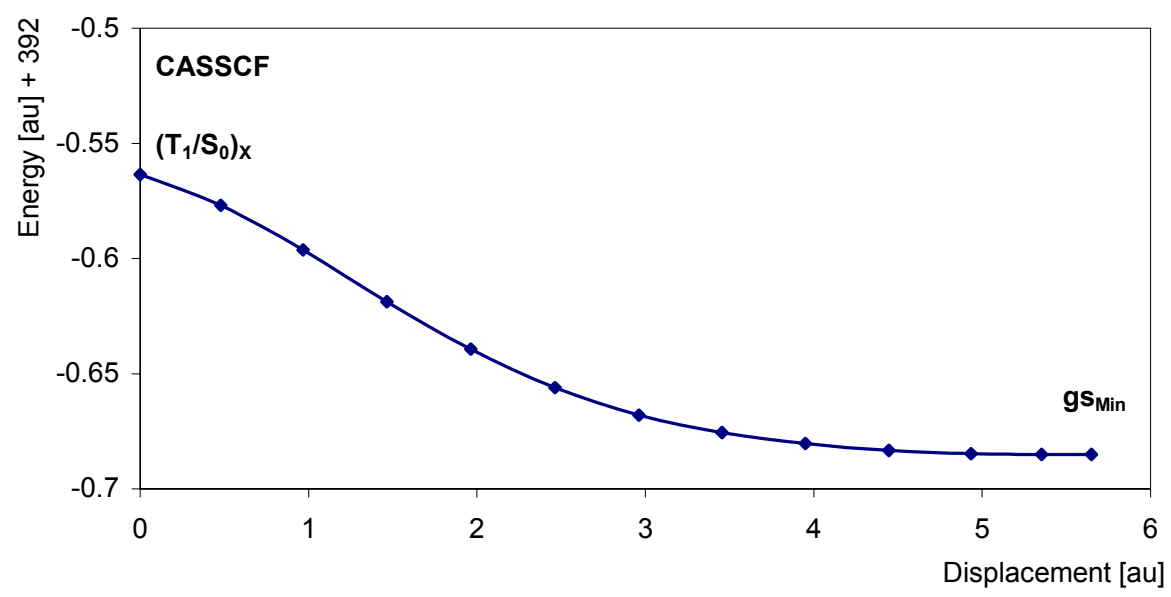

Figure SI11. Calculated MEP (CASSCF) from $\left(\mathbf{T}_{\mathbf{1}} / \mathbf{S}_{\mathbf{0}}\right)_{\mathbf{X}}$ to $(\mathbf{g s})_{\min }$. 